\title{
Design and Implementation of Small Target Detection System based on Invariant Feature
}

\author{
Ting Bai, Jinwen Tian, Xiao Sun \\ ${ }^{a}$ National Key Laboratory of Science \& Technology on Multi-spectral Information Processing, School \\ of Automation, Huazhong University of Science and Technology, Wuhan, 430074, China \\ abtingleo@gmail.com
}

Keywords: Image retrieval; invariant feature; target detection; mathematical morphology; Target Tracking

\begin{abstract}
In our study, computer graphics are discussed and they are collected by the image sensor with an analog electrical signal. During acquisition of image, 3D space is relative to the camera system of different viewpoints with the same goals, it will cause the target respect to the change in orientation of imaging system and attitude, which will change the target in the imaging system. Images showed the same target image. Change the viewpoint to target in 3D space, which relative to the movement of the imaging system, such as change of the target rotation, translation and scale. We need to find or construct some sort of goal in order to make the motion or the target image features do not change. Such features and feature amounts are called invariance, and invariants. It is invariance only reflect the same characteristics or the same type of target, or that the nature with differences between different modes in the visual information. Because they are in 3D space with the target location, attitude and independent according to people who are easy to find the right target.
\end{abstract}

\section{Introduction}

Modern technology has been able to use a variety of means to gather a lot of information and produce various types of multimedia data. With the rapid expansion of multimedia information on the Internet, more and more people are exposed to variety of multimedia information. Multimedia information in most importantly, is the most important visual information. Visual information includes image information, video information, image sequence information and computer graphics and animation information [1-4]. The largest multimedia information is the most important image information, an image-based study multimedia services has become a hot topic in information technology [5].

Content-based image retrieval system can retrieve the object which is divided into: related query system, query system and target classification inquiry system. In a related inquiry system, the inquirer at the start of query did not identify targets, such as the first retrieval system [6]. In the target system query, the query object find one given goal, which may be given in the query by example of figure exactly the same picture, it may be different images of the same object. Classified any given query is a query image and the image of the same kind. This means users often query is somewhat the same, and the image pickup tool, but external conditions lead to some different images. In order to achieve these goals retrieval purposes, it has been proposed image retrieval system based on invariant features.

\section{The key target detection technology based on invariant feature}

The principle of content-based image retrieval feature was shown in Fig. 1. The tissue image data retrieval system will be characterized according to the content analysis features of the image in figure establish content-based feature of a feature vector (or index). On this basis, index database structure and a pointer index associated with the actual image. When retrieved, the users in the system with the help of its own constitution by selecting an image to be retrieved or describe the color ratio, object shape, texture, or select the example of figure. Retrieval system using the same method to treat 
retrieved image feature analysis, retrieval feature type, feature indexing and retrieval library, indexed and retrieval those features characteristics sufficient formula with similar images retrieved [7, 8]. General search result was thumbnails, image URL or form URL of the page containing the image information appears.

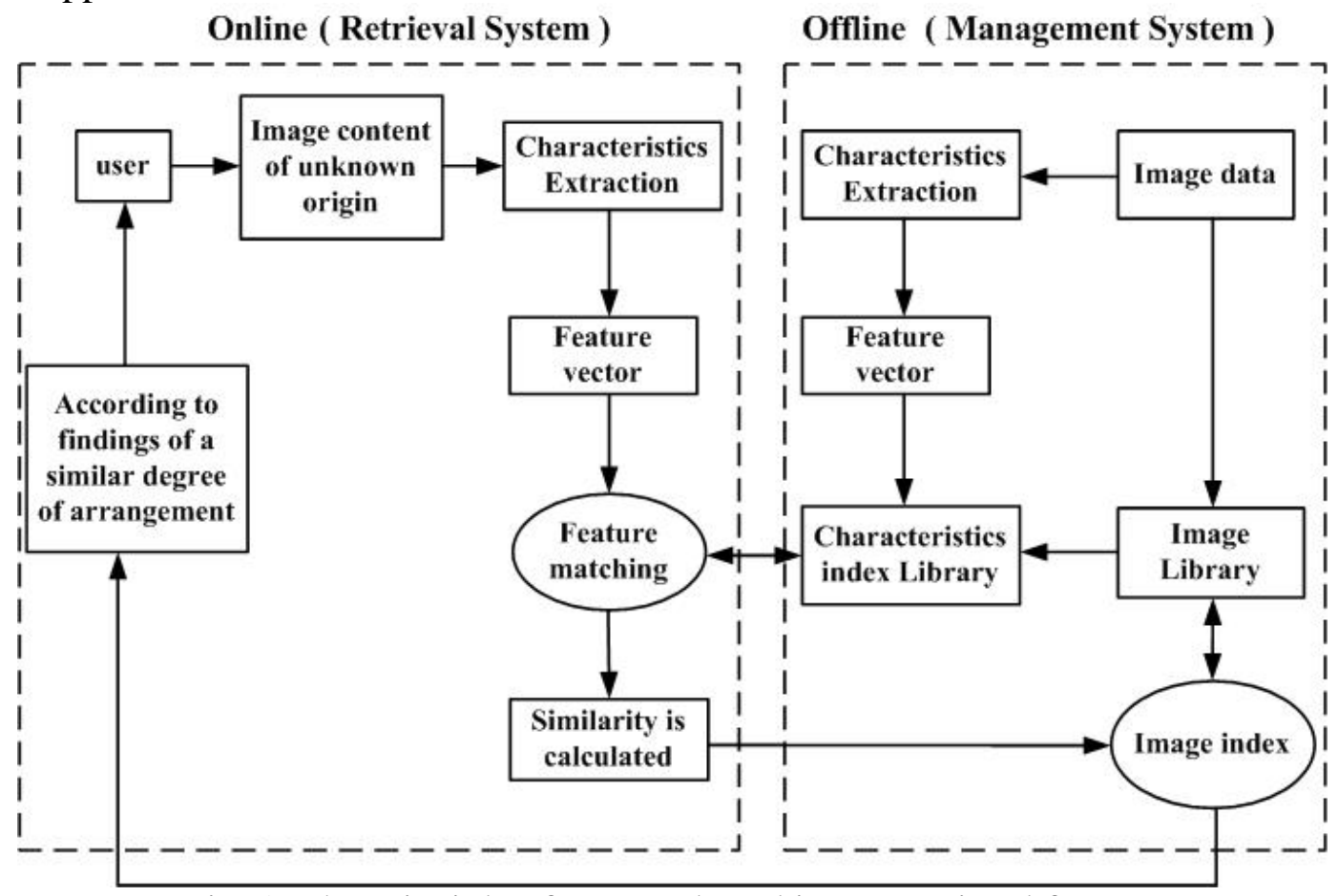

Fig. 1. The principle of content-based image retrieval feature

Extractions of the image content can establish the image database. In the image archive, the first input is analyzed to extract feature vector of the image or object. In the input image is stored in the database while the corresponding eigenvectors also credited with the signature image database linked. We analyze and extract their feature vectors. Characterized by the feature vector library feature matching, image database search the matching results. It can extract the desired image. Users can further browse the output, select the desired image and modify the query or advice based on the results. So we can see from the above works invariant feature based image retrieval technology. It has three key aspects: First, we must select the appropriate image feature, the second is to take effective feature extraction method, third is to have accurate feature matching algorithm $[9,10]$.

\section{Small target image features and model}

Most of the noises in images are statistically independent, that is, the infrared image can be considered noise distribution as Gaussian distribution:

$$
n(x, y)=\frac{1}{\sqrt{2 \pi \sigma}} \exp \left(-\frac{1}{2 \sigma^{2}}\right)
$$

Where $\sigma$ is the standard difference of the image.

Image pre-processing technology is a series of means for the image to improve the final effects and it is to make the image more suitable for human or machine for the methods of analysis and process of the next step. Image pre-processing has two basic elements: enhanced region of interest, suppress a region of interest. It did not have to approach the original image, without increasing the original image information. From the perspective of the entire computer vision classification, image pre-processing technologies include: grayscale correction, image smoothing, image sharpening, color enhancement, image correction. Wherein, the small target in infrared image areas commonly used technique mainly in image smoothing neighborhood. The main technical method is the mean, median, 
band-pass filtering. Currently classification academia common small target image preprocessing technology is spatial domain filtering and frequency domain filtering categories.

Assuming that $f(x, y)$ is the original input image, the difference image $m(x, y)$ is the value obtained by filtering the image, $\mathrm{s}(\mathrm{x}, \mathrm{y})$ values are the original image and the filtered image. $\mathrm{P}(\mathrm{x}, \mathrm{y})$ is the corresponding pixel $(\mathrm{x}, \mathrm{y})$ neighborhood $(\mathrm{N} \times \mathrm{N})$, then:

$$
\begin{gathered}
m(x, y)=\text { medfilter }[f(x, y)] \\
s(x, y)=f(x, y)-m(x, y)
\end{gathered}
$$

Fig. 2 are results of a small target image after adding noise by different sizes window of median filtering.

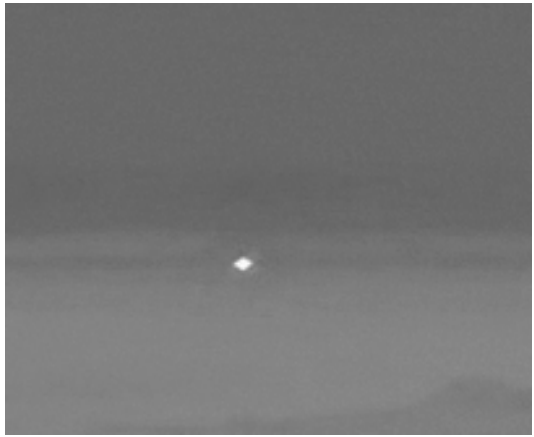

(a) Original image

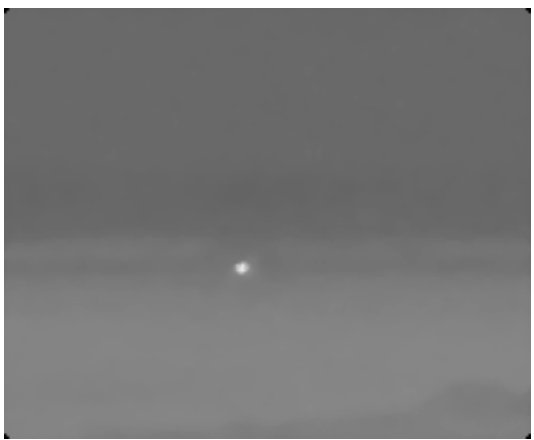

(c) $5 \times 5$ template

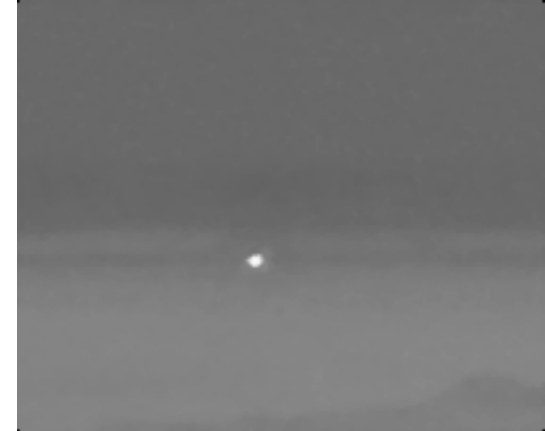

(b) $3 \times 3$ template

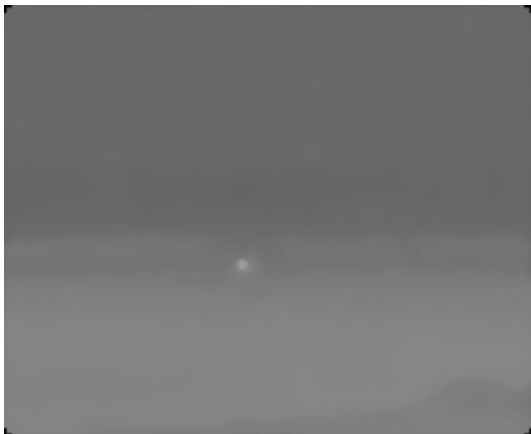

(d) $7 \times 7$ template

Fig. 2. Target with different sizes window of median filtering process

\section{Experiments and results}

In order to verify the feasibility and effectiveness, the algorithm triangular binary patterns match the template for target tracking. We conducted simulation experiments in Win7 platform Matlab software. Experiment showed the coefficient $\mathrm{k}$ takes the template 65, using $3 \times 3$ neighborhood binary mode, image sequence img1_350 img1_385. There is a big background and downs, lower image noise ratio because of a little target in infrared sequence. First frame of the image sequence is determined by the target image detecting method.

Comparing to $\mathrm{D}(\mathrm{x}, \mathrm{y}, \sigma)$ of local maxima and minima, each pixel with 8 points in the neighborhood to be up and down, it is layer adjacent smooth image in the neighborhood. If the pixel values are larger than the point of the pixel values surrounding it, it was chosen as the feature point. This process is shown in Fig. 3, the pixel values with a dotted circle in figure pixel values marked by stars from the surrounding points compared. 


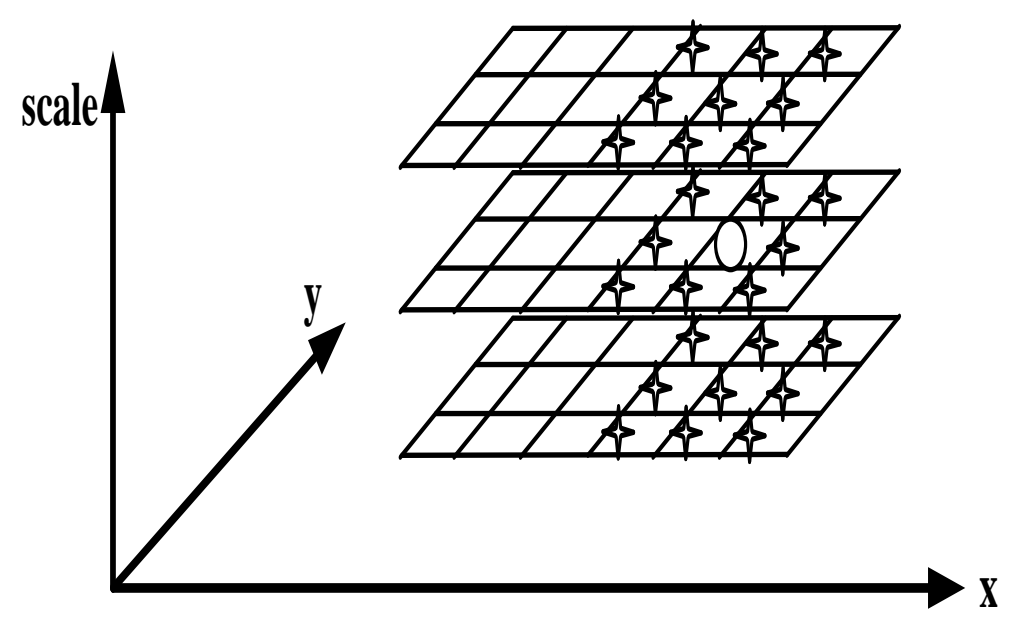

Fig. 3. The feature point extraction schematic

\section{Summary}

Invariant feature extraction pattern recognition has been an important research content. It currently used in image retrieval which is not mature. Invariant feature extraction algorithm applied mainly in moment invariant features, integral invariant features and invariant features. The moment invariant features often computationally intensive, and loss of local information of image. The image affects the distance, angle, resolution cameras and other factors. By showing the same object size, tilt and position, it will be different. Resulting in the existing feature extraction methods, it often failed to retrieve the desired effect. With the development of content-based image retrieval technology, image feature extraction for perspective remain the same, they are becoming increasingly important. According to the principle of perspective transformation, the image pick up viewing angle changes which caused rotation of translation and scales of the image plane with objective variety.

\section{References}

[1] Girshick R, Donahue J, Darrell T, et al. Rich feature hierarchies for accurate object detection and semantic segmentation[C]//Proceedings of the IEEE conference on computer vision and pattern recognition. 2014: 580-587.

[2] Kim S, Lee J. Scale invariant small target detection by optimizing signal-to-clutter ratio in heterogeneous background for infrared search and track[J]. Pattern Recognition, 2012, 45(1): 393-406.

[3] Dong X, Huang X, Zheng Y, et al. Infrared dim and small target detecting and tracking method inspired by human visual system[J]. Infrared Physics \& Technology, 2014, 62: 100-109.

[4] Garrido-Jurado S, Muñoz-Salinas R, Madrid-Cuevas F J, et al. Automatic generation and detection of highly reliable fiducial markers under occlusion[J]. Pattern Recognition, 2014, 47(6): 2280-2292.

[5] Tang L, Niemeijer M, Reinhardt J M, et al. Splat feature classification with application to retinal hemorrhage detection in fundus images[J]. Medical Imaging, IEEE Transactions on, 2013, 32(2): 364-375.

[6] Sun Q, Hu F, Hao Q. Mobile target scenario recognition via low-cost pyroelectric sensing system: Toward a context-enhanced accurate identification[J]. Systems, Man, and Cybernetics: Systems, IEEE Transactions on, 2014, 44(3): 375-384.

[7] Lin F, Dong X, Chen B M, et al. A robust real-time embedded vision system on an unmanned rotorcraft for ground target following[J]. Industrial Electronics, IEEE Transactions on, 2012, 59(2): 1038-1049.

[8] Yang Y, Newsam S. Geographic image retrieval using local invariant features[J]. Geoscience and Remote Sensing, IEEE Transactions on, 2013, 51(2): 818-832. 
[9] Jain A, Tompson J, LeCun Y, et al. Modeep: A deep learning framework using motion features for human pose estimation[M]//Computer Vision--ACCV 2014. Springer International Publishing, 2014: 302-315.

[10]Lee D, Kim G, Kim D, et al. Vision-based object detection and tracking for autonomous navigation of underwater robots[J]. Ocean Engineering, 2012, 48: 59-68. 